\title{
Next-Generation Maneuvering System with Control-Moment Gyroscopes for Extravehicular Activities Near Low-Gravity Objects
}

\author{
Michele Carpenter*, Kimberly Jackson, Babak Cohanim*, and Kevin R. Duda* \\ Draper Laboratory, Cambridge, Massachusetts, 02139, USA \\ Jared Rize $\dagger$ Celena Dopart $†$ and Jeffrey Hoffman ${ }^{\ddagger}$ \\ Massachusetts Institute of Technology, Cambridge, Massachusetts, 02139, USA
}

Pedro Curiel, Joseph Studak, Dina Poncia, and Jennifer Rochlis Zumbado ${ }^{\S}$

NASA Johnson Space Center, Houston, Texas, 77058, USA

\begin{abstract}
Looking ahead to the human exploration of Mars, NASA is planning for exploration of near-Earth asteroids and the Martian moons. Performing tasks near the surface of such lowgravity objects will likely require the use of an updated version of the Manned Maneuvering Unit (MMU) since the surface gravity is not high enough to allow astronauts to walk, or have sufficient resistance to counter reaction forces and torques during movements. The extravehicular activity (EVA) Jetpack device currently under development is based on the Simplified Aid for EVA Rescue (SAFER) unit and has maneuvering capabilities to assist EVA astronauts with their tasks. This maneuvering unit has gas thrusters for attitude control and translation. When EVA astronauts are performing tasks that require fine motor control such as sample collection and equipment placement, the current control system will fire thrusters to compensate for the resulting changes in center-of-mass location and moments of inertia, adversely affecting task performance. The proposed design of a next-generation maneuvering and stability system incorporates control concepts optimized to support astronaut tasks and adds control-moment gyroscopes (CMGs) to the current Jetpack system. This design aims to reduce fuel consumption, as well as improve task performance for astronauts by providing a stiffer work platform. The high-level control architecture for an EVA maneuvering system using both thrusters and CMGs considers an initial assessment of tasks to be performed by an astronaut and an evaluation of the corresponding human-system dynamics. For a scenario in which the astronaut orbits an asteroid, simulation results from the current EVA maneuvering system are compared to those from a simulation of the same system augmented with CMGs, demonstrating that the forces and torques on an astronaut can be significantly reduced with the new control system actuation while conserving onboard fuel.
\end{abstract}

\section{Introduction}

$\mathrm{T}$

HE feasibility of using control-moment gyroscopes (CMGs) in actuating a vehicle for EVAs was first demonstrated in the early 1970s with the M509 Skylab experiments, where a test bed maneuvering unit was operated from within Skylab and zero-gravity human performance data was analyzed. ${ }^{1}$ The Manned Maneuvering Unit (MMU) is a propulsive backpack that was designed at NASA Johnson Space Center (JSC) following the M509 tests. This system was widely used during the Space Shuttle era, when there were unprecedented opportunities for astronauts to perform extravehicular activity (EVA) tasks. ${ }^{2}$ The Simpified

\footnotetext{
*Technical Staff, 555 Technology Square, Cambridge, MA 02139.

$\dagger$ Graduate Research Assistant, 555 Technology Square, Cambridge, MA 02139.

$\ddagger$ Professor of the Practice, MIT Aero/Astro 37-227, 77 Massachusetts Avenue, Cambridge, MA 02139.

$\S$ Technical Staff, ER-411.
} 
Aid for EVA Rescue (SAFER) is a smaller, simplified version of the MMU designed for propulsive selfrescue in emergency situations where an EVA astronaut becomes detached from a safety tether and a shuttle is unable to perform a rescue. ${ }^{3-5}$ NASA JSC is currently developing a Jetpack device that evolves the SAFER concept into a maneuvering unit for astronauts to make repairs to inaccessible areas. ${ }^{6}$ This device also has applications for EVA tasks near the surface of low-gravity objects. Each of these maneuvering units uses cold-gas thrusters that respond to translation and attitude commands from an astronaut. Since these devices are mainly designed for approaching and capturing satellites, or for rescue maneuvers in the case of the SAFER, maintaining rigid attitude control to provide a stable work platform is not a design requirement. However, when EVA astronauts are attempting to perform tasks that require fine motor control such as sample collection and equipment placement near the surface of an asteroid or a Martian moon, the current control system will fire thrusters to compensate for the resulting changes in center-ofmass location and moments of inertia, adversely affecting task performance. This paper proposes to address this problem by augmenting the Jetpack device with a control-moment gyroscope (CMG) attitude control system. This Next-Generation (NextGen) Maneuvering Unit incorporates control concepts optimized for EVA task support. Since CMGs consume no propellant, this design also reduces fuel consumption over missions involving attitude compensation. Given sufficient electrical energy storage, using CMGs rather than thrusters for attitude control will extend the useful time of device operations between refueling.

Simulation results have been generated to evaluate the hypothesis that CMG attitude control improves stability, provides tighter attitude deadband control, and reduces consumables when added to the Jetpack device. The system simulation includes a human physics model that outputs torque as a function of input joint kinematics and human body parameters. An initial task assessment evaluates EVA task analyses from previous programs and determines the motions of interest. Section II discusses the existing Jetpack testbed and proposed next-generation system design. Section III describes the human model development and dynamics for EVA tasks of interest. Section IV discusses the system simulation environment, which includes the comparison of a CMG-augmented Jetpack system to the current thrusters-only configuration. This comparison study is performed for a specific CMG configuration and potential mission scenario with representative human dynamics from the GEBOD (Generator of Body Data) model.

\section{System Concept}

The system of interest for this study consists of the Jetpack device and the suited astronaut, shown below in Fig. 1(a). Attitude control and steering of the Jetpack is achieved by commanding thrust from combinations of 24 reaction control system (RCS) jets clustered in four main areas along the periphery of the structure. The Jetpack system responds to foot, voice, or computer-entered commands by the user. ${ }^{6}$ In the current engineering development unit setup shown in Fig. 1(b), there are two translational degrees of freedom and one rotational degree of freedom on an air-bearing floor. The device is connected to cold-gas tanks stored inside the Jetpack system.

The proposed design augments this system with an array of four single-gimbal CMGs and the electrical energy storage needed to power these devices. A single-gimbal CMG is a torque actuator consisting of a constant-speed rotor and a gimbal that changes the direction of the rotor's angular-momentum vector. The array of CMGs provides three-axis control with one level of redundancy for singularity avoidance. The design of the CMG attitude-control system and its integration into the current Jetpack is contingent on an assessment of EVA tasks along with the expected system dynamics.

\section{Human Model and Task Assessment}

EVA tasks of interest are evaluated using a human dynamics model that calculates system center of mass, moment of inertia, and torque profiles that can be integrated into the system simulation environment. Primary inputs include human body parameters and basic motions that are defined by the actuated joint kinematics. The development of these inputs, the Next-Generation Extravehicular Activity (NextGen EVA) human body model, and the human dynamics model are detailed in this section. 


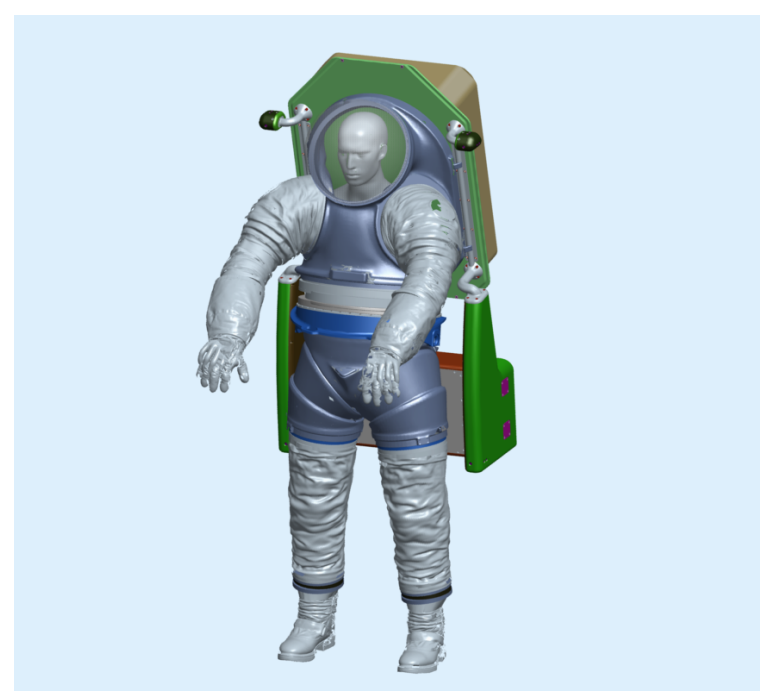

(a) Jetpack concept with suited astronaut.

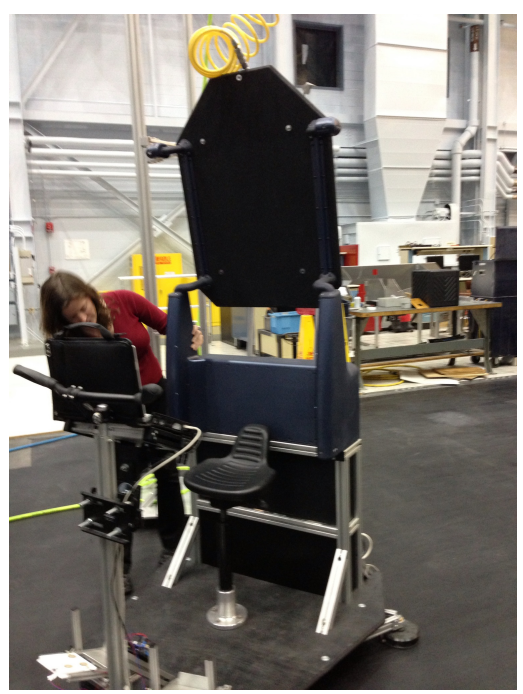

(b) Jetpack experimental setup.

Figure 1. Jetpack testbed.

\section{III.A. Body Parameters}

The unsuited astronaut body parameters are computed using the GEBOD program. ${ }^{7}$ The program calculates body segment geometric and mass properties based on subject's gender, height, and weight, specified either by percentile or user-input values. The model astronaut currently used for mechanical development of the Jetpack is a $180 \mathrm{lb}(81.6 \mathrm{~kg}), 72$ in $(1.83 \mathrm{~m})$ male. GEBOD uses these inputs to compute mass and inertial properties for the 14 segments of the NextGen EVA human body model.

The NextGen EVA human body model mimics the 17-segment GEBOD model and accounts for the astronaut's limited flexibility within the Extravehicular Mobility Unit (EMU), the pressurized space suit astronauts currently wear during EVAs. As shown in Fig. 2, GEBOD's 2, 3, 4, and 5 segments are combined into one main "torso" segment (L2) in the NextGen EVA human body model. The combination of these segments accounts for the rigidity of the torso, neck, and head of the EMU and restricts the independent mobility of the four segments. The human body models, with their respective numbering schemes, are illustrated in Fig. 2. $\hat{\mathbf{e}}_{i B}$ represents the basis vector along the $i$ direction in the Jetpack body fixed reference frame, $B$.

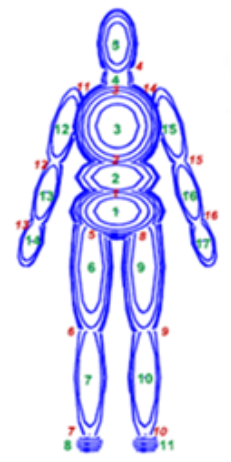

(a) GEBOD model.

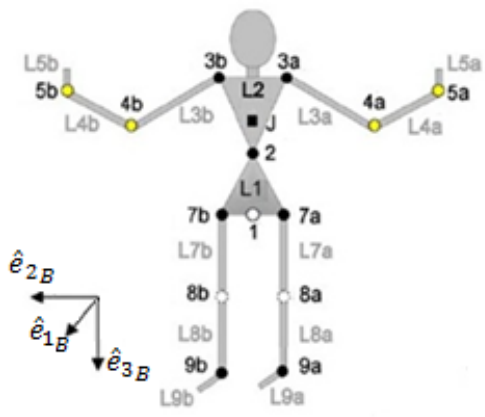

(b) NextGen EVA model (front view).

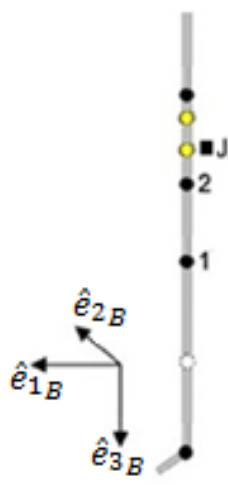

(c) NextGen EVA model (side view).

Figure 2. Human body model diagrams. 
The unsuited body parameters from GEBOD are augmented with mass properties of the $\mathrm{EMU}^{8}$ and the Jetpack assembly, and Table 1 details the combined EMU/Jetpack assembly part masses. These part masses are added to their associated segment mass and segment inertias are increased proportionately with mass increase. The center-of-mass locations are assumed unchanged for all segments except the torso, which is higher and further back due to the added mass and dimensions of the Jetpack assembly. Table 2 shows the augmented mass properties for each segment.

Table 1. EMU and Jetpack Assembly Mass Breakdown.

\begin{tabular}{lc}
\hline Part & Mass, kg \\
\hline Liquid Cooling and Ventilation Garment (LCVG) & 2.94 \\
Hard Upper Torso (HUT) & 12.29 \\
Arm Assembly & 7.87 \\
Lower Torso Assembly (LTA) & 20.72 \\
Gloves & 2.31 \\
Helmet & 8.23 \\
Jetpack Assembly & 119.30 \\
\hline
\end{tabular}

Table 2. Whole-System Mass Breakdown.

\begin{tabular}{lllc}
\hline Segment & Description & Associated Parts & Combined Mass, kg \\
\hline 2 & Upper Torso/Central & Helmet, HUT, Jetpack & 174.31 \\
& Torso/Head/Neck & Assembly, LCVG & \\
1 & Pelvis & LTA, LCVG & 17.51 \\
3a & Upper Arm & Arm Assembly, LCVG & 4.33 \\
4a2 & Lower Arm & Arm Assembly, LCVG & 3.04 \\
5a2 & Hand & Glove & 1.67 \\
3b & Upper Arm & Arm Assembly, LCVG & 4.33 \\
4b2 & Lower Arm & Arm Assembly, LCVG & 3.04 \\
5b2 & Hand & Glove & 1.67 \\
7a & Thigh & LTA & 15.17 \\
8a & Calf & LTA & 6.02 \\
9a & Foot & LTA & 1.49 \\
7b & Thigh & LTA & 15.17 \\
8b & Calf & LTA & 6.02 \\
9b & Foot & LTA & 1.49 \\
\hline
\end{tabular}

\section{III.B. Motion}

Along with the human body parameters, motion trajectories are required as inputs to the human dynamics model. A preliminary task list was compiled to include selected tasks likely to be performed during an asteroid EVA. The task list will provide necessary inputs for the astronaut dynamics model, including external forces and torques, mass properties of any corresponding tools, and definition of the motion trajectory. The task list will be organized by grouping similar tasks and motions together, and is expected to ultimately help pinpoint Jetpack control modes based on intended task type.

Limited information is available concerning tool specifications, corresponding motions, and external force and torque data; however, guidelines described here will be used to populate the task list and offer minimum and maximum torque limits for each task. The maximum external torque to which a crewmember can 
comfortably react while in free float is just under $70 \mathrm{Nm}$, and the maximum external force is just under 220 N. ${ }^{9}$ Impulses for each can be higher, but astronauts are generally instructed to avoid exceeding the limits. In terms of motion characteristics, arm movements will likely be the most common, given the type of motions seen on EVA (repairs, geological sampling, equipment deployment). Most arm motions remain within the immediate work envelope of the astronaut's chest, defined as roughly between the shoulders, between the eyes and belly button, and within the forward reach of the arms with a slight bend.

Since near-Earth asteroid (NEA) exploration is one of the EVA areas that would likely benefit from a CMG-stabilized Jetpack system, the initial task assessment is based on prior NEA mission analysis programs, specifically the NASA Extreme Environment Mission Operations (NEEMO) and Desert Research and Technology Studies (Desert RATS, or D-RATS). Missions from both programs include analyses of task procedures and tool use that primarily consist of geological sampling methods to meet the science objectives of NEA exploration. ${ }^{10}$ Between the two programs, three categories of sampling techniques were tested: surface sampling, soil sampling, and depth sampling. ${ }^{11}$ Surface sampling consists of hand, bag, or contact surface pad collection, soil sampling consists of clam-shell device or scoop collection, and depth sampling consists of core-tube or drive-tube collection. ${ }^{12}$ Surface sampling (e.g., picking up a rock) tends to have the lowest reaction forces, while depth sampling (e.g., deploying a drive tube into the ground) tends to have the highest. There are some exceptions; for example, a small surface sample may require a hammer to separate it from a larger rock, which creates a significantly higher impulse.

While specifications for the aforementioned sampling tools are not readily available, several items from ${ }^{3}$ have been chosen as representative EVA tools for this study. A hammer, a power drill, and a torque wrench have been selected to offer a range of external impulses, forces, and torques. Selected tool specifications and descriptions are included in Table 3 and shown in Fig. 3. A task list compiled from these tool specifications, the minimum and maximum guidelines described in the beginning of this section, and the task procedures learned from NEEMO and D-RATS missions provides a concise resource from which motion inputs for the astronaut dynamics code can be readily found.

Table 3. Tool Specifications and Descriptions.

\begin{tabular}{lccl}
\hline Tool & Mass, kg & Length, cm & Description \\
\hline Hammer & 0.91 & 27.94 & $\begin{array}{l}\text { Tool used for disconnect and jam removal with } \\
\text { fiberglass shaft and brass head to damp the } \\
\text { shock of hammer blows. } \\
\text { Battery-powered, two-speed/four-torque unit } \\
\text { with forward/reverse switch. Used for any } \\
\text { EVA task that requires a portable torque de- } \\
\text { vice. } \\
\text { Retractable ratchet wrench that applies be- } \\
\text { tween } 20.3 \text { to } 135.6 \mathrm{Nm}(15 \text { to } 100 \mathrm{ft}-1 \mathrm{~b}) \text { of } \\
\text { torque. }\end{array}$ \\
\hline
\end{tabular}

The following section includes an analysis of a simple motion input defined by actuated joint position, velocity, and acceleration. In this case, there are no corresponding tools, external forces, or external torques. In this motion, both arms swing upward from the neutral straight-down position to directly outward from the chest, and the speed of the motion is varied to demonstrate the resulting changes in the torque profile. Figure 4 depicts the initial and final body positions for the defined motion.

\section{III.C. Human Dynamics Model}

The human dynamics model provides the torque profile required to maintain stability during the various motions defined in the astronaut task list. The program is adapted from an existing code design that simulates astronaut self rotation. ${ }^{8}$ The 37-degree-of-freedom NextGen EVA human body model contains 14 chain-linked segments as shown in Fig. 2(b), with the pelvis (segment L1) as the base. Each segment consists of a point mass at the location of the segment's mass center, a length, and a corresponding moment of inertia. Segment reference frames are defined at the base joint of the corresponding segment; for example, 


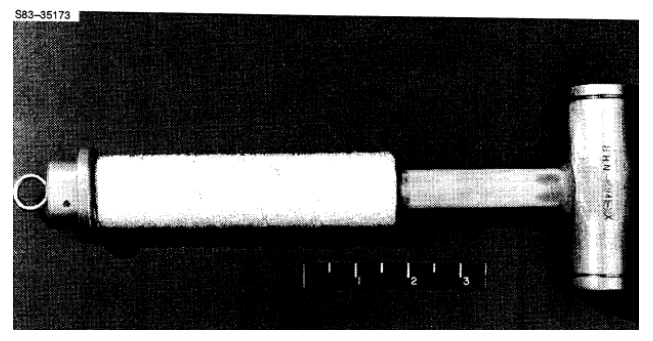

(a) Hammer.

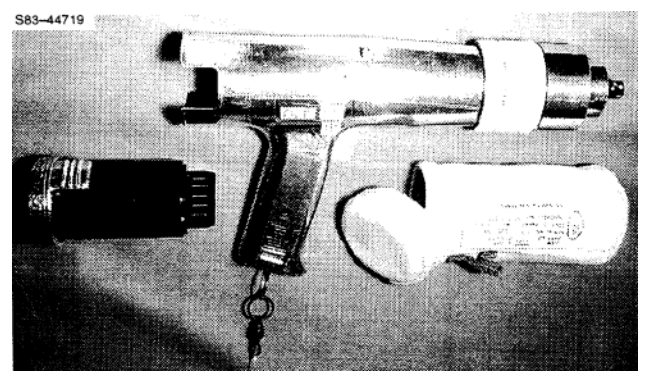

(b) EVA Power Tool.

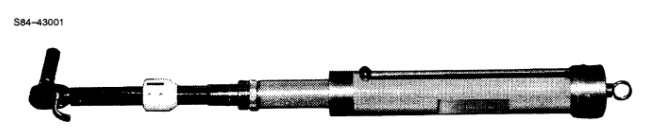

(c) Torque Wrench.

Figure 3. Selected tools.

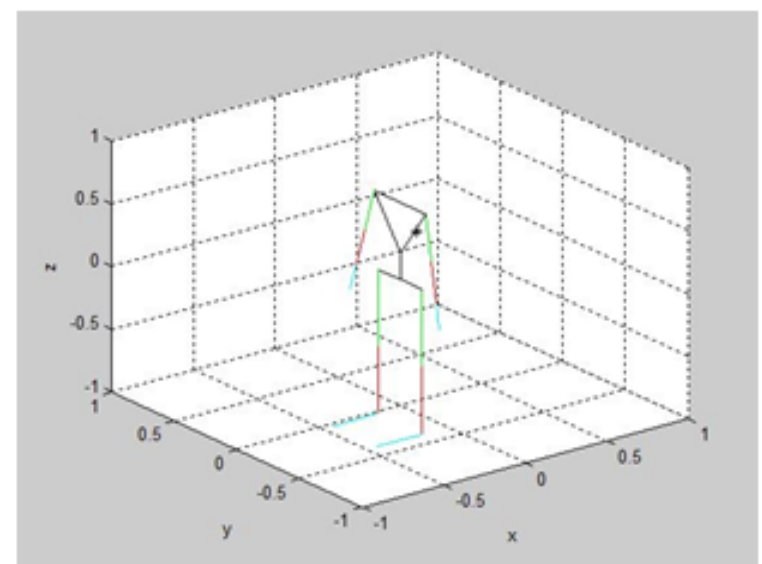

(a) Initial position.

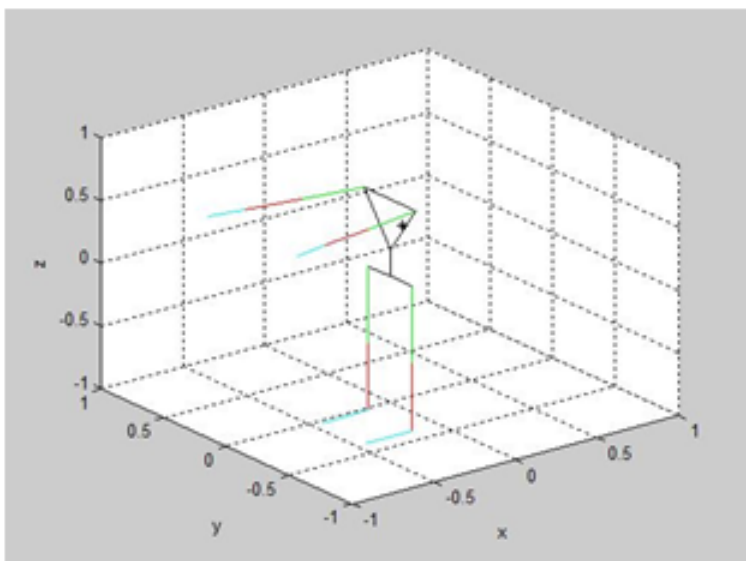

(b) Final position.

Figure 4. Body positions.

the reference frame for the pelvis, segment L1, has its origin at the base of the pelvis, joint 1 . This pelvis reference frame, or the 1-frame, is the frame from which whole-body properties, or system-level properties, are defined. Figures 2(b)-(c) show the location of the $B$-frame origin, denoted point J, in the NextGen EVA human body model. Point $\mathrm{J}$ is coincident with the whole-body center of mass in the neutral position where the legs are straight down and the arms are straight down by the sides.

The original astronaut self-rotation code ${ }^{8}$ uses a combination of forward and inverse dynamics to compute the desired mass, inertia, and torque properties. Any motion is initially defined by specifying actuated joint position, velocity, and acceleration. The trajectory of the unactuated joint connecting the body to the inertial frame is determined for the defined motion of the actuated joints, as well as the joint forces and torques required to create the actuated joint motions. For each motion, the body is held fixed with respect to the inertial frame. In other words, the actuated joint motion is identical to the unactuated joint motion and the astronaut does not rotate or translate as a result of the movement. The net torque about $\mathrm{J}$ required to maintain the fixed inertial position is computed, and the center-of-mass location and moments of inertia are calculated as the body position changes. These three outputs (torque, mass-center location, and moments 
of inertia) are computed in $B$ coordinates.

Three simple cases are executed using the motion described in the previous section and illustrated in Fig. 4. The three cases differ only in the length of time to complete the motion: the slowest at $10 \mathrm{~s}$ for Case 1 , $5 \mathrm{~s}$ for Case 2, and the fastest at $1 \mathrm{~s}$ for Case 3. The torque profile for each of these cases is shown in Fig. 5. As expected from the defined motion, the required torque is entirely about $\hat{\mathbf{e}}_{2 B}$, indicating that if the astronaut were unrestrained, raising the arms would cause the astronaut to tilt forward. Additionally, the maximum torque increases proportionally with the speed of the arms, from $0.64 \mathrm{Nm}$ for a $10 \mathrm{~s}$ motion to $6.43 \mathrm{Nm}$ for a $1 \mathrm{~s}$ motion. A torque profile for a particular motion is the input from the astronaut dynamics code to the CMG-augmented Jetpack system simulation.

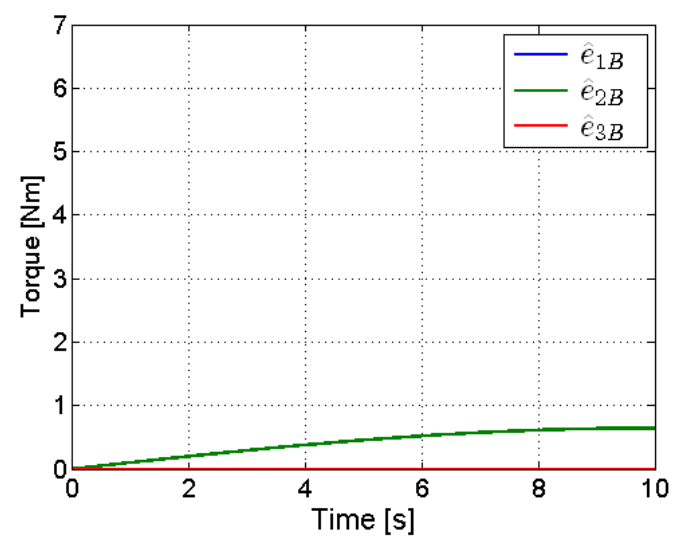

(a) Case 1: 10 s motion.

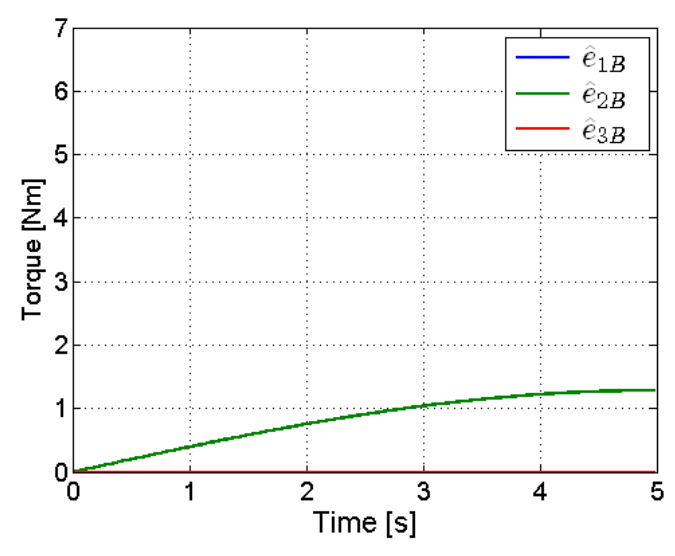

(b) Case 2: $5 \mathrm{~s}$ motion.

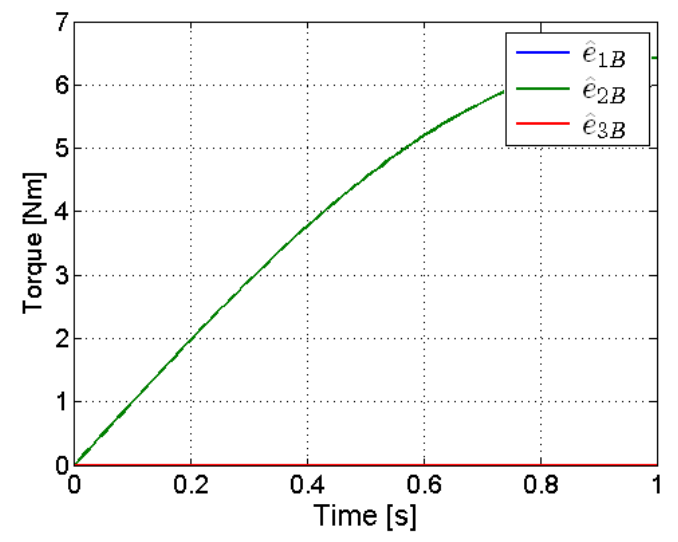

(c) Case 3: $1 \mathrm{~s}$ motion.

Figure 5. Torque about system mass center expressed in $B$ coordinates.

\section{System Simulation}

NASA's Multi-Mission Space Exploration Vehicle (MMSEV) closed-loop simulation ${ }^{13}$ provides the baseline for the Jetpack simulation environment. This simulation models RCS jet placement and orientation, as well as an array of four CMGs. The MMSEV simulation was selected for this study because of its existing architecture that could be readily adapted for the enhanced Jetpack system. The intent of this simulation tool is to evaluate representative EVAs and quantify the differences between the current and proposed system designs. For an example mission, fuel consumption and attitude stability are compared for the current and proposed system designs. 
The CMG-stabilized Jetpack simulation tool can execute several task plans, control modes, and pointing modes. The task plan refers to the vehicle's nominal trajectory, as defined in the asteroid-centered inertial (ACI) coordinate frame. The control mode refers to the active control system, which is responsible for both translational and rotational control inputs. The three control modes include CMGs only, jets only, and combined jets and CMGs. The CMGs-only mode is restricted to orbital task plans since it cannot provide $\Delta V$. Implementation and performance of the jets-only and combined jets/CMGs control modes are of primary interest and the focus of discussion in this section. There are three options for pointing mode, which refers to the vehicle's orientation during its trajectory: free drift, attitude hold, and target tracking. The free-drift mode does not provide any reference input to the control algorithms and can be used to simulate what would happen if the attitude-control system were to fail or if the vehicle ran out of propellant and/or electrical power. The attitude-hold pointing mode commands the same attitude throughout the mission such that the vehicle maintains the same pointing direction. For example, this mode would be used for sustaining an astronaut's pointing vector towards the center of an asteroid during orbit. An attitude hold would also be used for an EVA task where the astronaut maintains attitude in the presence of external disturbances, particularly reaction disturbances from performing the task. The final pointing mode is the target track mode which allows the astronaut to specify a primary and secondary target location in the ACI frame. Figure 7 shows the $B$ basis vectors relative to the Jetpack. The control algorithm aligns $\hat{\mathbf{e}}_{1 B}$ with the vector from the vehicle to the primary target as outlined in. ${ }^{13}$ The simulation environment allows the user to define task plan, control mode, and pointing mode. Parameters associated with the selected options are assigned during initialization.

Figure 6 shows the top-level Simulink block diagram of the closed-loop simulation environment. The simulation components are separated into categories such as actuators, satellite dynamics, sensors, GNC, and data logging. In order to compare the jets-only and combined control modes, the simulation includes control algorithms that allow these control modes to work both independently and cooperatively.

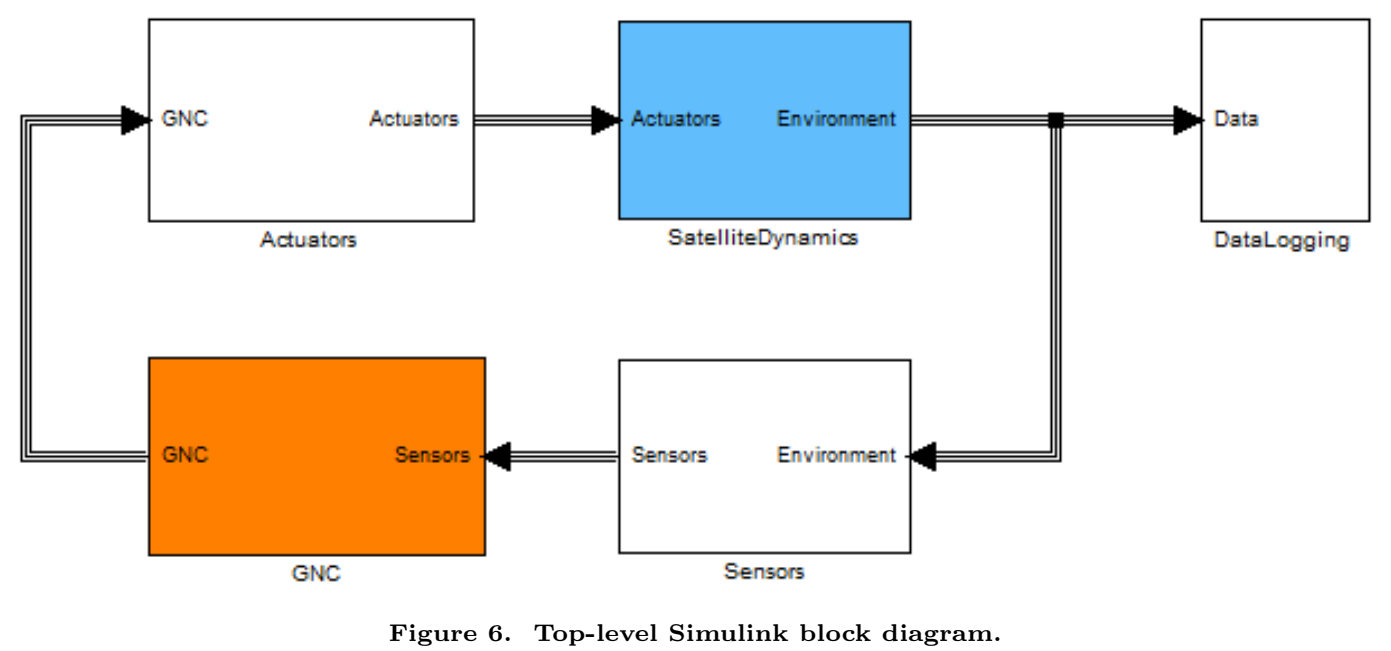

\section{IV.A. RCS Jet Control Algorithm}

The RCS jet system is comprised of 24 thrusters clustered in four primary areas of the Jetpack. The diagram in Fig. 7 shows the $B$ basis vectors, $\hat{\mathbf{e}}_{i B}$, and vectors conveying the direction of force acting on the vehicle (negative of the actual thrust direction). Table 4 indicates the thruster locations in $B$ coordinates measured from the system mass center.

The average mission length is based on total onboard fuel and the propellant's specific impulse, $I_{s p}$. The RCS thrusters, which use nitrogen gas, each nominally produce $0.8 \mathrm{lbf}(3.56 \mathrm{~N})$ with an $I_{s p}$ of $72 \mathrm{~s}$. The total onboard fuel mass is $6.9 \mathrm{lb}(3.13 \mathrm{~kg})$, which yields a total usable time of approximately 10.4 min assuming continuous single-thruster firing. Tridyne $\left(91 \% \mathrm{GN}_{2}, 6 \% \mathrm{H}_{2}\right.$ and $3 \% \mathrm{O}_{2}$ molar) is another propellant being considered for use with the Jetpack's RCS jets. Tridyne has an $I_{s p}$ of $135 \mathrm{~s}$, which yields approximately 19.4 min of continuous single-thruster firing. Equation 1 computes mission length, $t_{\max }$, in seconds from the $I_{s p}$ 


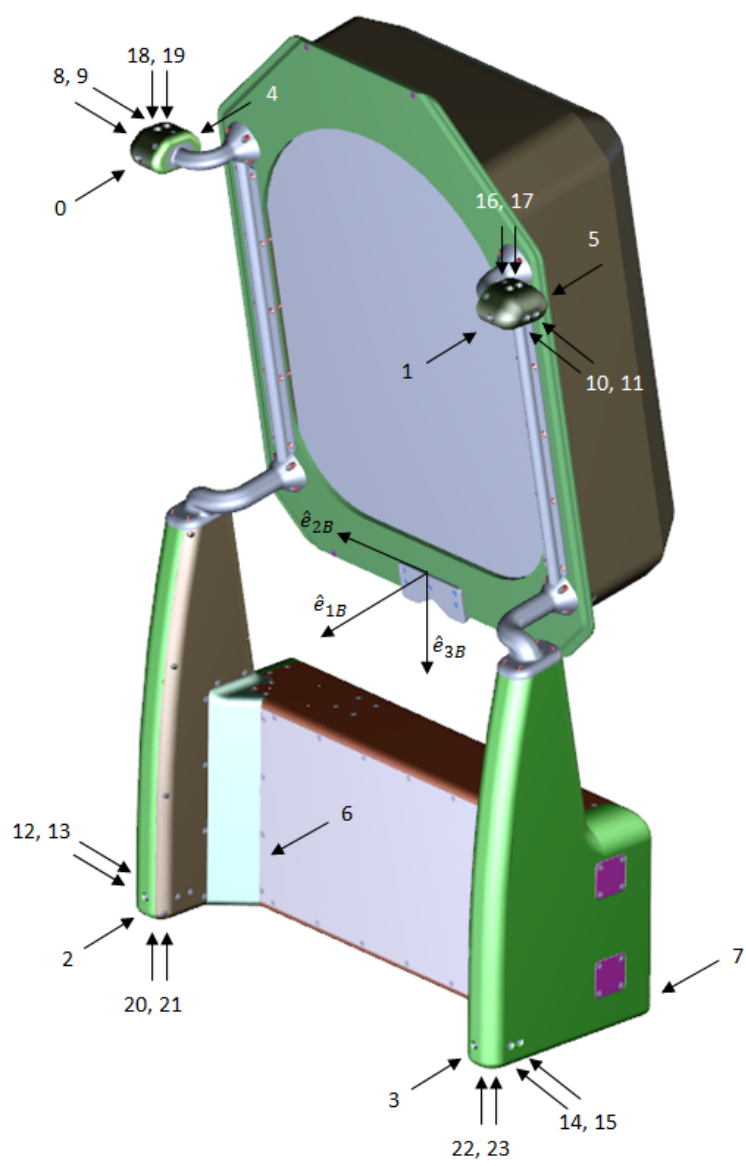

Figure 7. RCS jet locations and force vectors.

and total fuel

$$
t_{\max }=\frac{M}{F /\left(I_{s p} g_{0}\right)},
$$

where $F$ is the magnitude of force from a single thruster, $M$ is the total onboard fuel mass, and $g_{0}=9.81$ $\mathrm{m} / \mathrm{s}^{2}$.

Two RCS algorithms are investigated for use on this system: single-axis phase plane ${ }^{14}$ and revised simplex algorithm. ${ }^{15}$ The single-axis phase plane control algorithm is better known and can be modeled such that the system behaves as an on-off actuator by matching the controller frequency to the thruster's minimum on time. After initial implementation of the combined three-axis phase-plane controller, it was apparent that treating each axis independently and actuating the RCS jets to reduce the error was ineffective. Specifically, the vehicle's moment of inertia (MOI) about its mass center, expressed in $B$ coordinates, has large off-diagonal terms that introduce significant cross-coupling torques during thruster firings. Expressing the vehicle's thruster location and reference accelerations in the principal MOI frame $(P)$ would eliminate any off-axis accelerations from the thruster firings and no cross-coupling would occur. In order to conduct the phase-plane analysis in $P$ coordinates, the principal MOI and corresponding transformation matrix are found by diagonalizing the non-principal MOI tensor. The direction-cosine matrix from $B$ to $P$ coordinates, $T_{B}^{P}$, describes how the $i$ th basis vector in $P$ relates to the $i$ th basis vector in $B$ :

$$
{ }^{P} \hat{e}_{i B}=T_{B}^{P} \cdot{ }^{B} \hat{e}_{i B}
$$

In Eq. 2, the column matrix ${ }^{P} \hat{e}_{i B} \in \mathbb{R}^{3}$ is the projection of the vector $\hat{\mathbf{e}}_{i B}$ onto the basis vectors fixed in $P$ and ${ }^{B} \hat{e}_{i B} \in \mathbb{R}^{3}$ is the projection of the same vector onto the $B$-fixed basis vectors. The calculation in Eq. 2 
Table 4. RCS Jet Identification.

\begin{tabular}{crrrcc}
\hline Thruster & X, in & \multicolumn{1}{c}{ Y, in } & Z, in & Direction & ID \\
\hline 0 & 3.510 & 14.070 & -19.870 & $-\mathrm{X}$ & RTop3 \\
1 & 3.510 & -14.070 & -19.870 & $-\mathrm{X}$ & LTop3 \\
2 & 3.397 & 13.143 & 23.659 & $-\mathrm{X}$ & RBot3 \\
3 & 3.397 & -13.140 & 23.659 & $+\mathrm{X}$ & LBot3 \\
4 & -0.066 & 14.070 & -19.870 & $+\mathrm{X}$ & RTop4 \\
5 & -0.069 & -14.070 & -19.870 & $+\mathrm{X}$ & LTop4 \\
6 & -8.600 & 13.750 & 23.986 & $+\mathrm{X}$ & RBot4 \\
7 & -8.600 & -13.750 & 26.986 & $+\mathrm{X}$ & LBot4 \\
8 & 2.060 & 15.400 & -19.870 & $-\mathrm{Y}$ & RTop5 \\
9 & 1.380 & 15.400 & -19.870 & $-\mathrm{Y}$ & RTop6 \\
10 & 2.060 & -15.400 & -19.860 & $+\mathrm{Y}$ & LTop5 \\
11 & 1.380 & -15.400 & -19.860 & $+\mathrm{Y}$ & LTop6 \\
12 & 1.946 & 14.455 & 23.659 & $-\mathrm{Y}$ & RBot5 \\
13 & 1.267 & 14.455 & 23.659 & $-\mathrm{Y}$ & RBot6 \\
14 & 1.947 & -14.500 & 23.659 & $+\mathrm{Y}$ & LBot5 \\
15 & 1.267 & -14.500 & 23.659 & $+\mathrm{Y}$ & LBot6 \\
16 & 2.060 & -14.070 & -21.194 & $+\mathrm{Z}$ & RTop1 \\
17 & 1.383 & -14.070 & -21.194 & $+\mathrm{Z}$ & RTop2 \\
18 & 2.060 & 14.070 & -21.197 & $+\mathrm{Z}$ & LTop1 \\
19 & 1.383 & 14.070 & -21.197 & $+\mathrm{Z}$ & LTop2 \\
20 & 1.947 & 13.143 & 24.986 & $-\mathrm{Z}$ & RBot1 \\
21 & 1.267 & 13.143 & 24.986 & $-\mathrm{Z}$ & RBot2 \\
22 & 1.947 & -13.140 & 24.986 & $-\mathrm{Z}$ & LBot1 \\
23 & 1.267 & -13.143 & 24.986 & $-\mathrm{Z}$ & LBot2 \\
\hline & & & & &
\end{tabular}

yields a -45 deg rotation about $\hat{\mathbf{e}}_{3 B}$, illustrated in Fig. 8 .

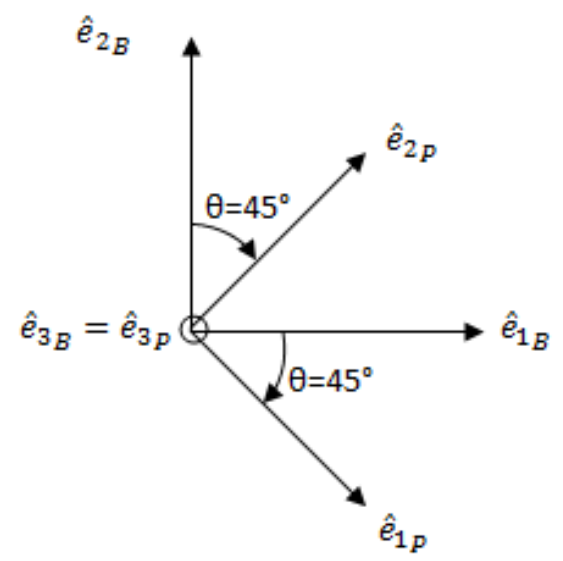

Figure 8. Rotation from vehicle body axes to principal axes.

A lookup table correlates how a jet on-time command in $P$ relates to the jet on-time command in $B$. The fact that the two frames differ by a $45 \mathrm{deg}$ rotation simplifies the complexity of the RCS lookup table. If the phase-plane controller, which is supplied as instantaneous acceleration data in $P$, outputs a jet on-time 
command about $\hat{\mathbf{e}}_{1 P}$, the lookup table provides the corresponding jet on-time command in $B$. Mapping from $P$ to $B$ coordinates for the jet on-time commands is a matter of vector addition:

$$
\begin{aligned}
& \hat{\mathbf{e}}_{1 P}=\hat{\mathbf{e}}_{1 B}-\hat{\mathbf{e}}_{2 B} \\
& \hat{\mathbf{e}}_{2 P}=\hat{\mathbf{e}}_{1 B}+\hat{\mathbf{e}}_{2 B} .
\end{aligned}
$$

For the example in Eqs. 3-4, a jet on-time command for positive $\hat{\mathbf{e}}_{1 P}$ is equivalent to simultaneous on-time commands for positive $\hat{\mathbf{e}}_{1 B}$ and negative $\hat{\mathbf{e}}_{2 B}$. The lookup table contains entries relating positive and negative on-time commands for the principal axes to the vehicle's body axes.

The revised simplex method determines jet on-time commands from $\Delta V$ and $\Delta \omega$ inputs. From an initial starting point, this linear programming method tests adjacent vertices of the feasible set, improving the objective cost until the optimal solution is reached. ${ }^{16}$ For the system of interest, this solution corresponds to the jet on-times required to achieve the $\Delta V$ and $\Delta \omega$ input commands.

These control algorithms are evaluated by comparing their performance (e.g., fuel consumption and attitude error) against one another during the same sample mission scenario. The sample mission consists of a one-dimensional burn (along Z-ACI frame) during which the Jetpack executes an attitude hold procedure. Table 6 summarizes the simulation results for each control algorithm in this scenario, demonstrating that the revised simplex algorithm produces the required jet on-time commands while minimizing fuel consumption.

The phase-plane analysis of the jets-only control mode in $P$ coordinates shows a better attitude error response, but requires a higher amount of fuel than the jets-only control mode in $B$ coordinates by a factor of 2 . As a result, the jet-select simplex algorithm is the attitude-control algorithm of choice for the jets-only control mode.

Table 5. Key Mission Parameters.

\begin{tabular}{ll}
\hline Parameter & Value \\
\hline Task plan & Octagon (discrete) \\
Pointing mode & Target tracking \\
Mission length & $4 \mathrm{hrs}$ \\
Onboard fuel mass & $3.12 \mathrm{~kg}$ \\
Initial roll error & $7 \mathrm{deg}$ \\
Initial velocity & Circular orbit velocity \\
Initial velocity error & $0 \mathrm{~m} / \mathrm{s}$ \\
\hline
\end{tabular}

Table 6. Simulation Results - RCS Control Algorithm Comparison

\begin{tabular}{lcccc}
\hline Algorithm & Fuel Usage, kg & $\begin{array}{l}\text { Max Roll } \\
\text { Error, deg }\end{array}$ & $\begin{array}{l}\text { Max Pitch } \\
\text { Error, deg }\end{array}$ & $\begin{array}{l}\text { Max Yaw } \\
\text { Error, deg }\end{array}$ \\
\hline Phase plane (in $P$ ) & 0.058 & 7.006 & 1.211 & 2.545 \\
Phase plane (in B) & 0.028 & 7.006 & 2.464 & 2.808 \\
Simplex (in B) & 0.029 & 7.063 & 1.556 & 1.920 \\
\hline
\end{tabular}

\section{IV.B. CMG Control Algorithm}

CMGs are widely studied for spacecraft attitude control and momentum management. Single-gimbal CMGs consist of a constant-speed rotor mounted on a gimbal that changes the direction of the rotor's constantmagnitude angular-momentum vector. CMGs are used for precision pointing applications since small gimbal torques can produce large output torques on the vehicle. The CMG controller accepts gimbal-rate commands as an input and imparts an output torque to the spacecraft. Since each CMG provides attitude control about 
a single axis, at least three CMGs are required (depending on the architecture) to achieve actuation for all three rotational degrees of freedom. Typically, at least one or more CMGs are also added to ensure that the control system has some level of fault tolerance. Much work has been done in characterizing different CMG configurations. Figure 9 defines the CMG pyramid configuration being tested in the Jetpack simulation environment. The pyramidal architecture consists of four CMGs with gimbal axes orthogonal to the faces of a pyramid. ${ }^{17-19} \mathbf{h}_{j}$ is the $j$ th CMG's angular-momentum vector and $\delta_{j}$ is the $j$ th CMG's gimbal angle. Each CMG gimbal axis is oriented with skew angle $\beta=54.73 \mathrm{deg}$ to achieve a spherical momentum envelope.

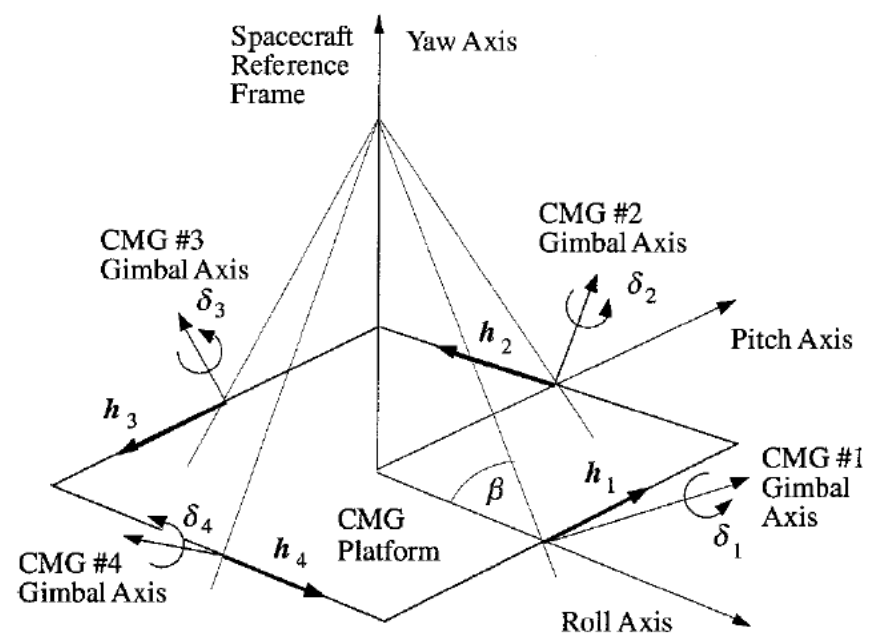

Figure 9. Pyramid CMG configuration. ${ }^{19}$

Three-axis attitude control is achieved by controlling the CMG gimbal rates. The angular momentum of the system about its mass center, $\mathbf{H}$, is the sum of the Jetpack's angular momentum and the angular momentum of the CMG array:

$$
\begin{aligned}
\mathbf{H} & =\mathbf{I}_{B} \cdot \boldsymbol{\omega}^{B / N}+\sum_{j=1}^{n} \mathbf{h}_{j} \\
& =\mathbf{I}_{B} \cdot \boldsymbol{\omega}^{B / N}+\mathbf{h}_{\mathrm{cmg}}
\end{aligned}
$$

where $\mathbf{h}_{\mathrm{cmg}}$ is the angular momentum of the CMG array, $\mathbf{I}_{B}$ is the inertia dyadic of the Jetpack about its mass center, $\boldsymbol{\omega}^{B / N}$ is the angular velocity of frame $B$ relative to inertial frame, $N$, and $n$ is the number of CMGs. The time derivative of Eq. 5 with respect to $N$ yields the total torque on the system about its mass center:

$$
\frac{{ }^{N} d}{d t} \mathbf{H}=\mathbf{I}_{B} \cdot \frac{{ }^{B} d}{d t} \boldsymbol{\omega}^{B / N}+\frac{{ }^{B} d}{d t} \mathbf{h}_{\mathrm{cmg}}+\boldsymbol{\omega}^{B / N} \times\left(\mathbf{I}_{B} \cdot \boldsymbol{\omega}^{B / N}+\mathbf{h}_{\mathrm{cmg}}\right) .
$$

The torque imparted by the CMGs to the Jetpack is

$$
-\boldsymbol{\tau}_{\mathrm{cmg}}=\frac{{ }^{B} d}{d t} \mathbf{h}_{\mathrm{cmg}}+\boldsymbol{\omega}^{B / N} \times \mathbf{h}_{\mathrm{cmg}} .
$$

The first term in Eq. 7, or the time derivative in $B$ of the total CMG angular momentum, is expressed as ${ }^{B} \dot{h}_{\text {cmg }} \in \mathbb{R}^{3 \times 1}$ when projected onto the $B$-fixed basis vectors. The negative sign before $\boldsymbol{\tau}_{\text {cmg }}$ reflects the fact that the torque applied to the system is a reaction to the gimbal torque imparted by the CMG. ${ }^{B} \dot{h}_{\mathrm{cmg}}$ is a function of the CMG gimbal angles, $\delta$ :

$$
{ }^{B} \dot{h}_{\mathrm{cmg}}=\frac{\partial\left({ }^{B} h_{\mathrm{cmg}}\right)}{\partial \delta} \frac{\partial \delta}{\partial t}=J(\delta) \dot{\delta}
$$


where $\dot{\delta} \in \mathbb{R}^{n}$ contains the CMG gimbal rates and $J(\delta) \in \mathbb{R}^{3 \times n}$ is the Jacobian matrix for the proposed CMG configuration. Equation 8 is modified to implement a pseudoinverse steering law ${ }^{19}$ for calculating gimbal rates:

$$
\dot{\delta}=J^{T}\left(J J^{T}+\lambda I\right)^{B} \dot{h}_{\mathrm{cmg}}=J^{\dagger}{ }^{B} \dot{h}_{\mathrm{cmg}} .
$$

$J^{\dagger} \in \mathbb{R}^{n \times 3}$ is the pseudoinverse of $J, I \in \mathbb{R}^{3 \times 3}$ is the identity matrix, and $\lambda \in \mathbb{R}$ is a scaling factor determined by the proximity of the CMG array to a singular configuration. ${ }^{20}$

\section{IV.C. Control-Mode Comparison}

The sample mission, summarized in Table 5, is used to compare the control modes of interest: jets only and combined jets/CMGs. The translational motion for both control modes are equivalent during the mission, but the vehicle's attitude time histories show differences that are further described in this section. Simulation results for fuel consumption and attitude error are used to evaluate the overall performance of each control mode. Perfect sensor data is assumed for this comparison.

Figure 10 shows the Jetpack's nominal trajectory relative to an asteroid. The vehicle travels from the starting location, indicated by the green marker, to the ending location, indicated by the red marker. Both the start and end points of the trajectory are specified in the X-Z plane of the ACI frame. The trajectories can be defined either parametrically with a prescribed curve or discretely by choosing specific points in the ACI frame. The targeting algorithm used for these missions has been adapted from previous work on the Lambert boundary-value problem. ${ }^{21}$

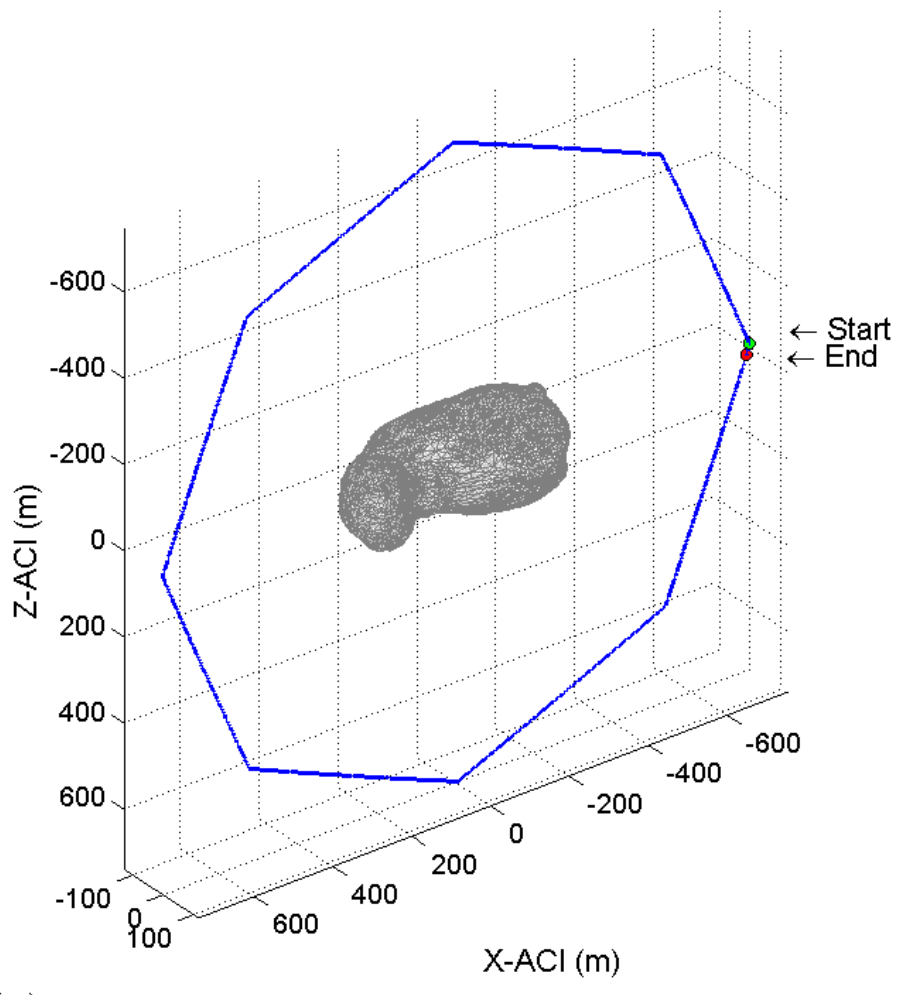

$\mathrm{Y}-\mathrm{ACl}(\mathrm{m})$

Figure 10. Nominal system trajectory relative to asteroid.

The simulation results in Figs. 11-12 indicate how the combined control mode consumes less fuel during the mission and maintains a smaller attitude error as expected. Fuel consumption for the jets-only simulation is much higher in Fig. 11 since the jets are responsible for both translation and attitude control. Since the jets-only mode has an on-off ability with a discrete minimum on-time, the jets cannot achieve a zero steadystate attitude error. This phenomenon is typically referred to as limit cycling. ${ }^{22}$ The attitude-control system recognizes that there is an error and fires the corresponding jets to counter the error; however, the minimum 
on-time of the jets causes the Jetpack to overcompensate. The minimum on-time of the jets is directly affected by physical properties of the system such as the opening and closing of valves, as well as softwarein-the-loop (SWIL) considerations such as the time required for computations and the amount of data being transferred. CMGs, on the other hand, are continuous control actuators with the ability to more significantly reduce attitude error. Figure 12 demonstrates that the CMGs are able to null the initial attitude error in each axis within $1 \mathrm{~min}$.

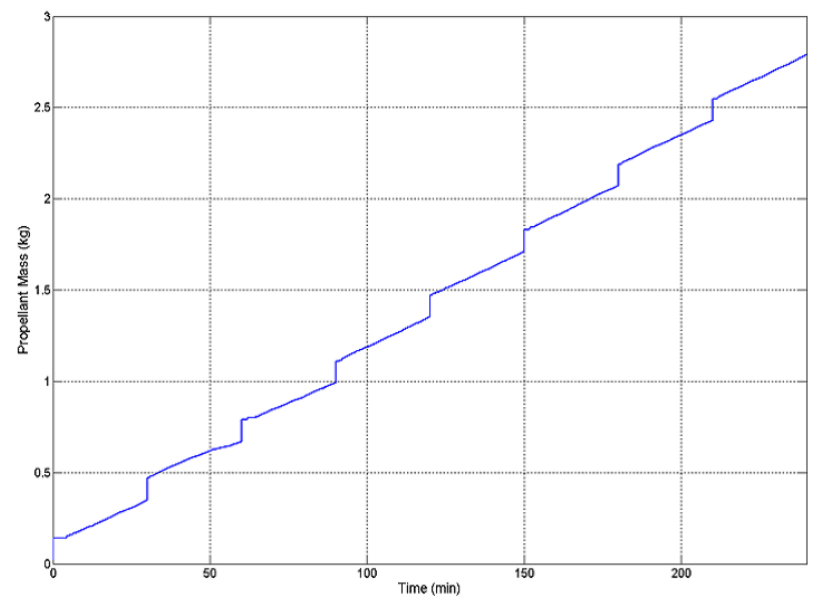

(a) Jets only.

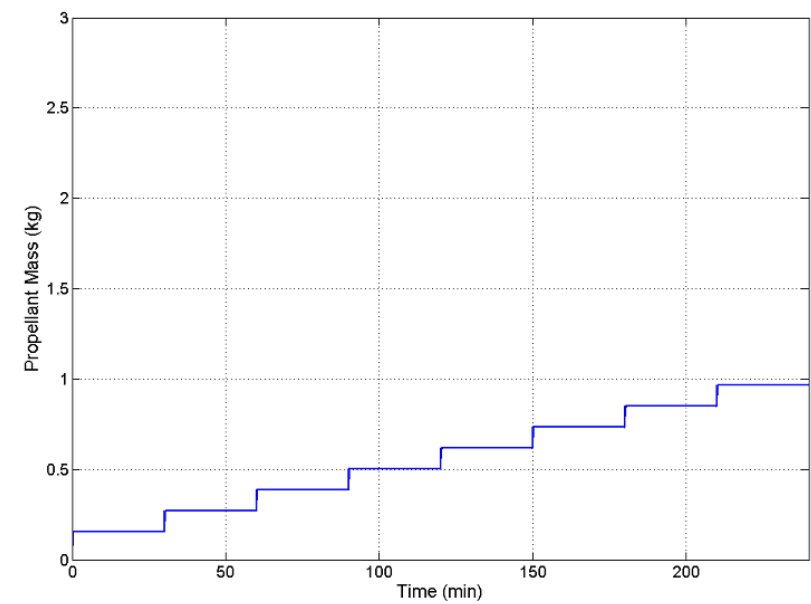

(b) Combined jets/CMGs.

Figure 11. Fuel consumption vs. time.
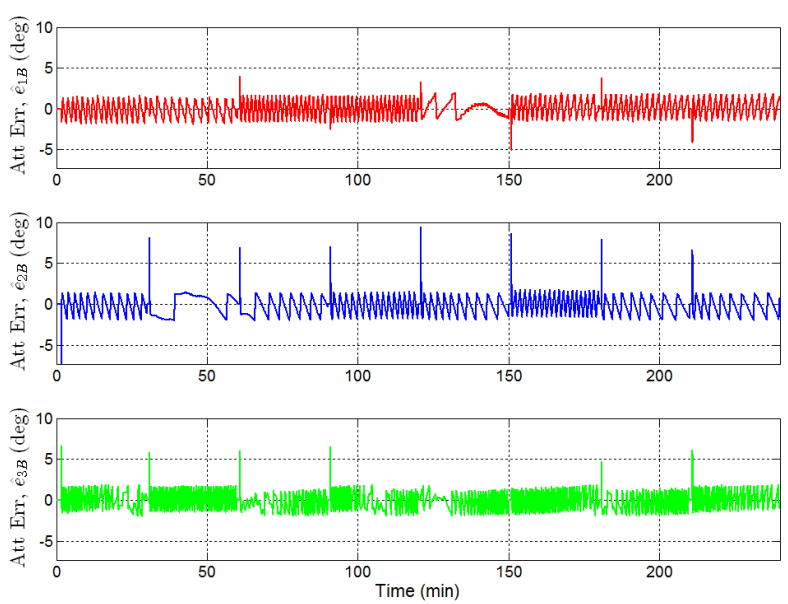

(a) Jets only.
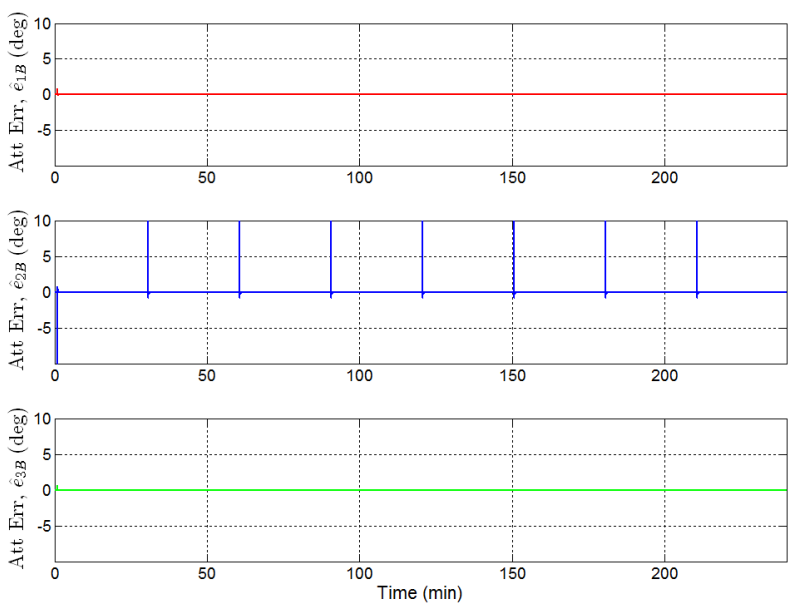

(b) Combined jets/CMGs.

Figure 12. Attitude error vs. time.

The smoother response of the CMGs is particularly important when considering the Jetpack's primary objective, which is to explore near-Earth asteroids and perform science-related tasks near the surface of low-gravity objects. The collection of samples from an asteroid's surface using a drill or hammering device could impart reaction forces and torques onto the free-floating astronaut, resulting in undesired translating or tumbling. While both control modes would return the astronaut back to the initial reference attitude, the CMGs would provide a smoother response, providing a stiffer work platform so that tasks can be performed without the use of multiple anchor points. Reducing the number of attachment points can greatly increase the astronaut's capability to work in unfamiliar areas, decreasing the amount of time needed to complete tasks. The combined control mode also consumes less fuel than the jets-only mode, demonstrated by a $65.95 \%$ reduction in fuel consumption for the combined control mode. 
The mass required to complete an EVA is the performance metric when comparing the proposed and current systems. To improve the current jets-only system design, the mass of the CMG array and accompanying energy-storage devices must be less than the fuel savings multiplied by the number of runs performed during an EVA. The $1.84 \mathrm{~kg}$ fuel savings for the scenario presented in this section scaled by number of runs during the EVA mission determines the upper bound for the mass of the entire CMG attitude-control system. With a CMG design based on a finalized task assessment, the mission will be re-evaluated to determine the more efficient system design.

\section{Conclusions and Future Work}

This study demonstrates that the current Jetpack device can be augmented with CMGs to provide greater attitude stability to EVA astronauts while conserving fuel. With a stiffer work platform, EVA tasks can also be performed more efficiently, which may further reduce the time required for task completion. For the human study, upcoming work will focus on the continued development of a task list based on selected tools. For the system simulation, alternate CMG configurations will be explored and the torque limits for tasks in the finalized task list will be used to appropriately size the CMGs and modify the control algorithm for mitigating CMG array saturation. The trade-space exploration of the different attitude-control systems (combined CMGs/jets and jets-only systems) will also include an evaluation of power usage for appropriate battery sizing. With a finalized design for the CMG-augmented Jetpack, the dynamics and control models will be integrated into the Virtual Reality Laboratory at NASA JSC, which currently provides a flight simulator for the SAFER. In addition to the planned quantitative analyses, performing simulated EVAs in this environment with a virtual-reality helmet and haptic-feedback devices will qualitatively demonstrate the benefits of the CMG-augmented Jetpack.

\section{References}

\footnotetext{
${ }^{1}$ Murtagh, T. B., Whitsett, C. E., Goodwin, M. A., and Greenlee, J. E., "Automatic Control of the Skylab Astronaut Maneuvering Research Vehicle," Journal of Spacecraft and Rockets, Vol. 11, No. 5, May 1974, pp. 321-326.

${ }^{2}$ Lenda, J. A., "Manned Maneuvering Unit: User's Guide," NASA-CR-151864, May 1978.

${ }^{3}$ Fullerton, R. K., "EVA Tools and Equipment Reference Book," Simplified Aid for EVA Rescue, NASA Johnson Space Center, JSC-20466 Rev. B, Nov. 20, 1993.

${ }^{4}$ Williams, T. and Baughman, D., "Self-Rescue Strategies for EVA Crewmembers Equipped with the SAFER Backpack," NASA Goddard Space Flight Center, Flight Mechanics/Estimation Theory Symposium, No. N94-35633, May 1, 1994, pp. $357-371$.

${ }^{5}$ Vassigh, K., Weigel, D., and Mack, T., "USA Simplified Aid for EVA Rescue (SAFER) Operations Manual," NASA Johnson Space Center, Systems Division, EVA Systems Group, JSC-26283 Rev. A, Aug. 20, 1998.

${ }^{6}$ Zumbado, J. R., Curiel, P. H., and Schreiner, S., "Hands-Free Control Interfaces for an Extra Vehicular Jetpack," Proceedings of the IEEE Aerospace Conference, Big Sky, Montana, March 2013.

${ }^{7}$ Cheng, H., Obergefell, L., and Rizer, A., "Generator of Body Data (GEBOD) Manual," General Dynamics Corporation, No. AL/CF-TR-1994-0051, March 1994.

${ }^{8}$ Stirling, L. A., "Development of Astronaut Reorientation Methods: A Computation and Experimental Study," Ph.D. Dissertation, Department of Aeronautics and Astronautics, Massachusetts Institute of Technology, 2008.

${ }^{9}$ Montalvo, E. J., Personal Communication, Jan. 24, 2013.

${ }^{10}$ Chappell, S. P., Abercromby, A. F., and Gernhardt, M. L., "NEEMO 15: Evaluation of Human Exploration Systems for Near-Earth Asteroids," NASA Johnson Space Center, GLEX-2012.06.1.6x12286, 2012.

${ }^{11}$ Chappell, S. P. and Abercromby, A. F., Personal Communication, Oct. 25, 2012.

${ }^{12}$ Abell, P., Personal Communication, Oct. 30, 2012.

${ }^{13}$ Jackson, M. C. and McDonald, R. D., "Draper Simulation Analysis Tool (DSAT): Graphical Object Simulation Techniques and Tools for Simulink," Proceedings of the AIAA Modeling and Simulation Technologies Conference and Exhibit, No. 20045458, Providence, Rhode Island, Aug. 2004.

${ }^{14}$ Johnson, M. C., "A Parameterized Approach to the Design of Lunar Lander Attitude Controllers," Proceedings of the AIAA Guidance, Navigation, and Control Conference, No. 2006-6564, Keystone, Colorado, Aug. 2006.

${ }^{15}$ Griva, I., Nash, S. G., and Sofer, A., Linear and Nonlinear Optimization, Society for Industrial and Applied Mathematics, Philadelphia, Pennsylvania, 2nd ed., 2009.

${ }^{16}$ Bazaraa, M. S., Jarvis, J. J., and Sherali, H. D., Linear Programming and Network Flows, John Wiley and Sons, Inc., Hoboken, New Jersey, 4th ed., 2010.

${ }^{17}$ Bedrossian, N. S., Paradiso, J., Bergmann, E. V., and Rowell, D., "Steering Law Design for Redundant Single-Gimbal Control Moment Gyroscopes," Journal of Guidance, Control, and Dynamics, Vol. 13, No. 6, Nov. 1990, pp. $1083-1089$.

${ }^{18}$ Paradiso, J. A., "Global Steering of Single Gimballed Control Moment Gyroscopes Using a Directed Search," Journal of Guidance, Control, and Dynamics, Vol. 15, No. 5, Sept. 1992, pp. 1236-1244.
} 
${ }^{19}$ Wie, B., Bailey, D., and Heiberg, C., "Singularity Robust Steering Logic for Redundant Single-Gimbal Control Moment Gyros," Journal of Guidance, Control, and Dynamics, Vol. 24, No. 5, Sept. 2001, pp. 865-872.

${ }^{20}$ Nakamura, Y. and Hanafusa, H., "Inverse Kinematic Solutions with Singularity Robustness for Robot Manipulator Control," Journal of Dynamic Systems, Measurement, and Control, Vol. 108, Sept. 1986, pp. 163-171.

${ }^{21}$ Battin, R. H. and Vaughan, R. M., "An Elegant Lambert Algorithm," Journal of Guidance, Control, and Dynamics, Vol. 7, No. 6, 1984, pp. 662-670.

${ }^{22}$ Jeon, S. W. and Jung, S., "Hardware-in-the-Loop Simulation for the Reaction Control System Using PWM-Based Limit Cycle Analysis," IEEE Transactions on Control Systems Technology, Vol. 20, No. 2, March 2012, pp. 538-545. 